\title{
A Type of Alternant
}

\author{
By F. W. Ponting
}

(Received 4th May, 1950. Read 3rd November, 1950.)

\section{Introduction.}

We define

$$
\alpha_{j}^{(k)}=\left(\alpha_{j}+\beta_{1}\right)\left(\alpha_{j}+\beta_{2}\right) \ldots\left(\alpha_{j}+\beta_{k}\right)
$$

where $\alpha_{p} \neq \alpha_{q}$ when $p \neq q$. If $N=\Sigma \lambda_{i}$, then the partition $\left(\lambda_{1}, \lambda_{2}, \ldots, \lambda_{n}\right)$ of $N$ with $\lambda_{1} \geqslant \lambda_{2} \geqslant \ldots \geqslant \lambda_{n}$ is denoted by $(\lambda)$ and we set

$$
l_{j}=\lambda_{j}+n-j \text {. }
$$

All partitions will be in descending order and the usual notation for repeated parts will be used.

The determinant with $f(s, t)$ in row $s$ and column $t$ will be denoted by $|f(s, t)|$. The use of $s$ and $t$ implies that the determinant is of order $n$. For other orders $\sigma$ and $\tau$ will be used.

We consider the function

$$
\{\alpha ;(\lambda) ; \beta\}
$$

defined by

If every $\beta_{i}=0$, then we have the $S$-function $\{\alpha ;(\lambda)\}$ defined by

[1, chap. VI].

$$
\left|\alpha_{s}^{l_{l}}\right|=\{\alpha ;(\lambda)\}\left|\alpha_{s}^{n-t}\right|
$$

When $0<v \leqslant u$, we define $b(u, v)$ to be the $v$-th elementary symmetric function of $\beta_{1}, \beta_{2}, \ldots, \beta_{u}$. We set $b(0,0)=1=b(u, 0)$ and $b(u, v)=0$ if $v<0$ or $u<v$. We take $H(u, v)$ as the $v$-th complete homogeneous symmetric function of $\beta_{1}, \beta_{2}, \ldots, \beta_{u}$ when $0<v$, and $H(0,0)=H(u, 0)=1$, $H(u, v)=0$ if $v<0$.

In this note we prove the following theorems:

Theorem 1. If $b\{(l),(r)\}=\left|b\left(l_{s}, l_{s}-l_{t}+r_{t}\right)\right|$, then

$$
\{\alpha ;(\lambda) ; \beta\}=\Sigma\left\{\alpha ;\left(\lambda_{1}-r_{1}, \lambda_{2}-r_{2}, \ldots, \lambda_{n}-r_{n}\right)\right\} b\{(l),(r)\},
$$

where the summation is taken over all non-negative $r_{i}$ such that

$$
\lambda_{1}-r_{1} \geqslant \lambda_{2}-r_{2} \geqslant \ldots \geqslant \lambda_{n}-r_{n} \geqslant 0 \text {. }
$$


TheOREM 2. If $\lambda_{1} \leqslant n,(\mu)$ and $\left(\mu-r^{\prime}\right)$ are partitions conjugate to $(\lambda)$ and $(\lambda-r)$ respectively and $m_{s}=\mu_{s}+n-s$, then

$$
b\{(l),(r)\}=\left|H\left(2 n-m_{s}, m_{s}-m_{t}+r_{i}{ }^{\prime}\right)\right|=H\left\{(m),\left(r^{\prime}\right)\right\}, \text { say. }
$$

THEOREM 3. The function $b\{(l),(r)\}$ may be expanded as a polynomial in $\beta_{1}, \beta_{2}, \ldots, \beta_{l_{1}}$ with positive integral coefficients.

Since Theorem 2 is solely concerned with the $\beta_{i}$, we can choose $n \geqslant \lambda_{1}$ by adding a sufficient number of zero parts to $(\lambda)$.

Hirsch [2] considered the case of $(\lambda)=\left(1^{n-k}, 0^{k}\right)$ and his result may be put in the form ${ }^{1}$

$$
\left\{\alpha ;\left(1^{n-k}, 0^{k}\right) ; \beta\right\}=\sum_{r=0}^{n-k}\left\{\alpha ;\left(1^{n-k-r}\right)\right\} H(k+1, r) .
$$

We may obtain the dual result

$$
\left\{\alpha ;\left(n-k, 0^{n-1}\right) ; \beta\right\}=\sum_{r=0}^{n-k}\{\alpha ;(n-k-r)\} b(2 n-k-1, r)
$$

by subtracting appropriate multiples of the columns of

$$
\left|\alpha_{s}^{\left(l_{l}\right)}\right|, \quad\left(l_{1}=2 n-k-1, l_{2}=n-2, l_{3}=n-3, \ldots, l_{n}=0\right),
$$

from the preceding columns.

Using Theorem 1, we find that in the expansion of (1) we have a term with

$$
\begin{gathered}
\lambda_{1}=\lambda_{2}=\ldots=\lambda_{n-k}=1, \quad \lambda_{n-k+1}=\lambda_{n-k+2}=\ldots=\lambda_{n}=0 \\
r_{1}=r_{2}=\ldots=r_{i}=0, \quad r_{t+1}=r_{i+2}=\ldots=r_{n-k}=1, \quad r_{n-k+1}=\ldots=r_{n}=0
\end{gathered}
$$

so that

$$
(\lambda)=\left(1^{n-k}\right), \quad(\lambda-r)=\left(1^{\ell}\right)
$$

and in Theorem 2

$$
(\mu)=(n-k), \quad\left(\mu-r^{\prime}\right)=(t) .
$$

Thus the coefficient of $\left\{\alpha ;\left(1^{t}\right)\right\}$ is

where

$$
b\{(l),(r)\}=\left|H\left(2 n-m_{s}, m_{s}-m_{t}+r_{l}^{\prime}\right)\right|
$$

$$
\begin{aligned}
& m_{1}=2 n-k-1, \quad m_{2}=n-2, \quad m_{3}=n-3, \quad \ldots, \quad m_{n}=0, \\
& r_{1}{ }^{\prime}=n-k-t, \quad r_{2}{ }^{\prime}=r_{3}{ }^{\prime}=\ldots=r_{n}{ }^{\prime}=0 .
\end{aligned}
$$

The first column of $\left|H\left(2 n-m_{s}, m_{s}-m_{t}+r_{t}^{\prime}\right)\right|$ now has

$$
H(k+1, n-k-t)
$$

in the first row and zero below, since $m_{3}-m_{1}+r_{1}{ }^{\prime}<0$ for $s>1$. The other columns will have unity on the principal diagonal position and zero below. Hence we have (1).

1 We omit zero parts when there is no danger of ambiguity. 
In (2), we find that we have a term with

$$
\lambda_{1}=n-k, \quad r_{1}=r, \quad \lambda_{j}=r_{j}=0 \quad(j>2),
$$

and the coefficient of $\left\{\alpha ;\left(1^{n-k-r}\right)\right\}$ is $b(2 n-k-1, r)$ from Theorem 1 .

As a further example we consider

$$
\left|\begin{array}{lll}
\alpha_{1}^{(4)} & \alpha_{1}^{(2)} & 1 \\
\alpha_{2}^{(4)} & \alpha_{2}^{(2)} & 1 \\
\alpha_{3}^{(4)} & \alpha_{3}^{(2)} & 1
\end{array}\right|=\left|\begin{array}{ccc}
\alpha_{1}^{2} & \alpha_{1} & 1 \\
\alpha_{2}^{2} & \alpha_{2} & 1 \\
\alpha_{3}^{2} & \alpha_{3} & 1
\end{array}\right|\{\alpha ;(2,1,0) ; \beta\} .
$$

We denote $b(u, v)$ by $u, v,\{\alpha ; \lambda\}$ by $\{\lambda\},-t$ by $\bar{t}$, and find that

$$
\begin{aligned}
& \{\alpha ;(2,1,0) ; \beta\}=\{2,1\}\left|\begin{array}{lll}
4,0 & 4,2 & 4,4 \\
2, \overline{2} & 2,0 & 2,2 \\
0, \overline{4} & 0, \overline{2} & 0,0
\end{array}\right|+\{2\}\left|\begin{array}{lll}
4,0 & 4,3 & 4,4 \\
2, \overline{2} & 2,1 & 2,2 \\
0, \overline{4} & 0, \overline{1} & 0,0
\end{array}\right| \\
& +\left\{1^{2}\right\}\left|\begin{array}{lll}
4,1 & 4,2 & 4,4 \\
2, \overline{1} & 2,0 & 2,2 \\
0, \overline{3} & 0, \overline{2} & 0,0
\end{array}\right|+\{1\}\left|\begin{array}{lll}
4,1 & 4,3 & 4,4 \\
2, \overline{1} & 2,1 & 2,2 \\
0, \overline{3} & 0, \overline{1} & 0,0
\end{array}\right|+\left|\begin{array}{lll}
4,2 & 4,3 & 4,4 \\
2,0 & 2,1 & 2,2 \\
0, \overline{2} & 0, \overline{1} & 0,0
\end{array}\right| \\
& =\{2,1\}+\{2\} b(2,1)+\left\{1^{2}\right\} b(4,1)+\{1\} b(4,1) b(2,1)+\left|\begin{array}{ll}
b(4,2) & b(4,3) \\
b(2,0) & b(2,1)
\end{array}\right| .
\end{aligned}
$$

As an example of Theorem 3, we consider

$$
\left|\begin{array}{ccc}
4,2 & 4,3 & 4,4 \\
2,0 & 2,1 & 2,2 \\
1, \overline{1} & 1,0 & 1,1
\end{array}\right|=b\{(4,2,1) ;(2,1,1)\} .
$$

This is the term independent of the $\alpha_{i}$ in the expansion of $\left\{\alpha ;\left(2,1^{2}\right) ; \beta\right\}$ and it does not factorise into determinants of the same type but lower order. The term independent of the $\alpha_{i}$ in the expansion of $\left\{\alpha ;\left(1^{3}\right) ; \beta\right\}$ also has this property and it is $h(1,3)$. These two terms are the first of order 3 which have the property.

Now

$$
\begin{aligned}
& b\{(4,2,1),(2,1,1)\} \\
& =\left|\begin{array}{ccc}
\beta_{1} \beta_{2}+\left(\beta_{1}+\beta_{2}\right)\left(\beta_{3}+\beta_{4}\right)+\beta_{3} \beta_{4} & \beta_{1} \beta_{2}\left(\beta_{3}+\beta_{4}\right)+\left(\beta_{1}+\beta_{2}\right) \beta_{3} \beta_{4} & \beta_{1} \beta_{2} \beta_{3} \beta_{4} \\
1 & \beta_{1}+\beta_{2} & \beta_{1} \beta_{2} \\
0 & 1 & \beta_{1}
\end{array}\right|
\end{aligned}
$$

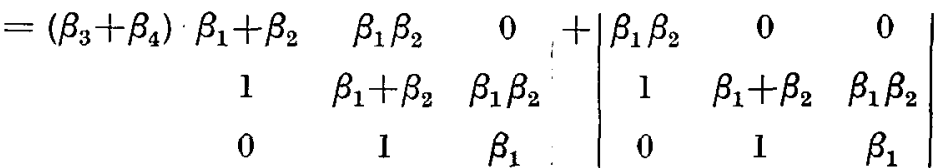

$$
\begin{aligned}
& =\left(\beta_{3}+\beta_{4}\right) \beta_{1}{ }^{3}+\beta_{1}{ }^{3} \beta_{2} \text {. }
\end{aligned}
$$


Since this note was first submitted, Foulkes [8] has given a different method of obtaining (1), (2) and Theorem 1.

2. Expansion of $\{\alpha ;(\lambda) ; \beta\}$.

We have, when $k \leqslant l_{1}$,

$$
\alpha_{j}^{(k)}=\sum_{i=k-l_{1}}^{k} b(k, i) \alpha_{j}^{k-i},
$$

so that $\left|\alpha_{s}^{\left(l_{s}\right)}\right|$ is the determinant of the product of the matrices

$$
A=\left[\alpha_{s}^{l_{1}-\tau}\right] \text { and } B=\left[b\left(l_{l}, l_{l}-l_{1}+\sigma\right)\right],
$$

where $\sigma, \tau=0,1,2, \ldots, l_{1} ; s, t=1,2, \ldots, n$.

It is well-known $[c f .3,86]$ that the determinant $|A B|$ is the sum of the $\left(\begin{array}{c}l_{1}+1 \\ n\end{array}\right)$ products of pairs of corresponding $n$-th order determinants which can be formed from $A$ and $B^{\prime}$, the transpose of $B$, each determinant occurring once only.

The determinant in $B^{\prime}$ corresponding to $\left|\alpha_{s}^{l_{t}-r_{t}}\right|$ is

$$
\left|b\left(l_{s}, l_{s}-l_{t}+r_{t}\right)\right| \text {. }
$$

We may select, and account for all $n$-th order determinants from $A$, by demanding that

$$
l_{1}-r_{1}>l_{2}-r_{2}>\ldots>l_{n}-r_{n}, \text { i.e. } \lambda_{1}-r_{1} \geqslant \lambda_{2}-r_{2} \geqslant \ldots \geqslant \lambda_{n}-r_{n} .
$$

Moreover, $r_{i} \leqslant \lambda_{i}$ since $l_{i}-r_{i} \geqslant n-i$, the least possible exponent for column $i$. Hence the coefficient of $\left\{\alpha ;\left(\lambda_{1}-r_{1}, \lambda_{2}-r_{2}, \ldots, \lambda_{n}-r_{n}\right)\right\}$ in the expansion of $\{\alpha ;(\lambda) ; \beta\}$ is

$$
\left|b\left(l_{s}, l_{s}-l_{l}+r_{t}\right)\right| \text {. }
$$

We have $l_{h} \leqslant l_{g}$ and $l_{g}-l_{j}+r_{j} \leqslant r_{g}$ for $j \leqslant g \leqslant h$. Hence, if $r_{g}<0$, then $b\left(l_{h}, l_{h}-l_{j}+r_{j}\right)=0$. In this case (3) vanishes, having zero elements in the first $g$ terms of the last $n-g+1$ rows. This completes the proof of Theorem 1 .

If $r_{o-1}<l_{g-1}-l_{g}$, i.e. $r_{g-1} \leqslant \lambda_{g-1}-\lambda_{g}$, we can show similarly that (3) factorises into two lower order determinants of the same kind as (3). We note that the graph $[1,67]$ of the partition $\left(\lambda_{1}-r_{1}, \lambda_{2}-r_{2}, \ldots, \lambda_{n}-r_{n}\right)$ must be regular for a non-zero term. However, if we construct this graph by removing the last $r_{i}$ nodes from row $i$ of the graph of $(\lambda)$ for $i=1,2, \ldots, n$ in succession, and if we have a regular graph at any, except the lest, stage, then (3) will factorise. 


\section{Duality.}

Lemma 1. The $p$-th order matrices $H_{1}=[H(p-\tau+1, \tau-\sigma)]$ and $B_{1}=\left[(-1)^{\tau-\sigma} b(p-\sigma, \tau-\sigma)\right]$ are reciprocal and hence adjoint.

Proof. If $H=[H(p, \tau-\sigma)], B=\left[(-1)^{\tau-\sigma} b(p, \tau-\sigma)\right]$, then $H B=I$, the unit matrix $[c f .3,115]$. We set $Q_{u r}$ as the $p$-th order square matrix with 1 on the principal diagonal, $-\beta_{u}$ in row $r-1$ of column $r$, and zero elsewhere,

Now

$$
Q_{u}=Q_{u, p} Q_{u, p-1} \ldots Q_{u, p-u+2} \text { and } Q=Q_{p} Q_{p-1} \ldots Q_{2}
$$

$$
\begin{aligned}
b(i+1, j+1) & =b(i, j+1)+\beta_{i+1} b(i, j), \\
H(i+1, j+1) & =H(i, j+1)+\beta_{i+1} H(i+1, j) .
\end{aligned}
$$

Then since $H(r, 0)=b(r, 0)=1=b(0,0)$, wè have $H Q=H_{1}$ and $Q^{-1} B=B_{1}$. Hence $H_{1} B_{1}=I$, and since the determinant of $H_{1}$ is 1 , then $H_{1}$ and $B_{1}$ are adjoint.

LEMma 2. If $\left(\lambda_{1}, \lambda_{2}, \ldots, \lambda_{n}\right)$ and $\left(\mu_{1}, \mu_{2}, \ldots, \mu_{n}\right)$ are conjugate partitions, then $n+s-\lambda_{s}$ and $n+1+\mu_{s}-s(s=1,2, \ldots, n)$, form a permutation of $1,2, \ldots, 2 n$.

This is merely a re-statement of Aitken's rule [5], that

$$
\left(\lambda_{n}, \lambda_{n-1}+1, \ldots, \lambda_{1}+n-1\right) \text { and }\left(\mu_{n}, \mu_{n-1}+1, \ldots, \mu_{1}+n-1\right)
$$

form bicomplementary sets in relation to the set $0,1,2, \ldots, 2 n-1$.

Proof of Theorem 2. This is based on a similar proof in [5]. If

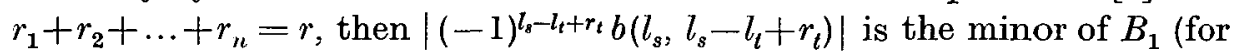
$p=2 n$ ) formed by rows $n+s-\lambda_{s}$ and columns $n+t-\left(\lambda_{t}-r_{t}\right)$, which by Jacobi's theorem and Lemma 2 , is equal to $(-1)^{r}$ times the minor formed from the transpose of $H_{1}$ by rows $n+1+\mu_{s}-s$ and columns $n+1+\mu_{t}-r_{l}{ }^{\prime}-t$. Hence we have

$$
\begin{aligned}
\left|b\left(l_{s}, l_{s}-l_{t}+r_{t}\right)\right|=(-1)^{r}\left|(-1)^{l_{s}-l_{t}+r_{t}} b\left(l_{s}, l_{s}-l_{t}+r_{t}\right)\right| \\
\quad=\left|H\left\{2 n+1-\left(n+1+\mu_{s}-s\right), n+1+\mu_{s}-s-\left(n+1+\mu_{t}-r_{t}{ }^{\prime}-t\right)\right\}\right| \\
\quad=\left|H\left(2 n-m_{s}, m_{s}-m_{t}+r_{t}{ }^{\prime}\right)\right| .
\end{aligned}
$$

This completes the proof of Theorem 2.

We note that from Lemma 1 we may deduce modified Wronski recurrence formulae; for $1 \leqslant r \leqslant p-1$, we have

$$
\begin{aligned}
& H(p, 0) b(p-1, r)-H(p-1,1) b(p-2, r-1) \\
& \quad+H(p-2,2) b(p-3, r-2)-+\ldots=0, \\
& b(p-1,0) H(p-r, r)-b(p-1,1) H(p-r, r-1) \\
& +b(p-1,2) H(p-r, r-2)-+\ldots=0 .
\end{aligned}
$$


If we replace $b(u, r)$ by $H(2 n-u, r)$ in $b\{(l),(r)\}$, we obtain

$$
\left|H\left(2 n-l_{s}, l_{s}-l_{t}+r_{t}\right)\right|
$$

which is equal to

$$
\left|b\left(m_{s}, m_{s}-m_{l}+r^{\prime}\right)\right| \text {, }
$$

thus illustrating the duality.

As in Section 2, we find that $H\left\{(m),\left(r^{\prime}\right)\right\}$ will factorise if

$$
r_{g-1}^{\prime}<m_{g-1}-m_{g}, \quad \text { i.e. } \quad r_{g-1}^{\prime} \leqslant \mu_{g-1}-\mu_{g} .
$$

Hence if we remove $r_{i}^{\prime}$ nodes from column $i$ of the graph of $(\lambda)$ in succession, and if, at any stage except the last, we have a regular graph, then $H\left\{(m),\left(r^{\prime}\right)\right\}$ will factorise into two determinants of the same type but of lower order.

4. Proof of Theorem 3.

We assume that

$$
r_{o-1} \geqslant l_{o-1}-l_{g} \quad(g=2,3, \ldots, n)
$$

so that $b\{(l),(r)\}$ does not factorise.

When $0<q \leqslant l_{u-1}-l_{u}$, we define

$$
\left(l_{u-1}, l_{u} \gamma q\right)
$$

to be the $q$-th elementary symmetric function of

$$
B_{l_{u+1}}, B_{l,+2}, \ldots, B_{l_{t-1}} \text {. }
$$

We set $\left(l_{u-1}, l_{u} \chi 0\right)=1$ and $\left(l_{u-1}, l_{u} \gamma r\right)=0$ if $r<0$ or $r>l_{u-1}-l_{u}$.

We denote the $n$-th order determinant

$$
\left|\begin{array}{l}
b\left(l_{u}, l_{u}-l_{t}+r_{t}+v_{s^{\prime}}\right) \\
\ldots \ldots . . . . . . . . . . . . . . . \\
b\left(l_{s^{\prime \prime}}, l_{s^{\prime \prime}}-l_{t}+r_{t}\right)
\end{array}\right| \quad\left(s^{\prime}=1,2, \ldots, u-1 ; s^{\prime \prime}=u, u+1, \ldots, n\right)
$$

by

\section{Now}

$$
\left[v_{1}, v_{2}, \ldots, v_{u-1}\right]
$$

$$
b\left(l_{u}, l_{u}-l_{t}+r_{t}\right)=\sum_{p=0}^{l_{u}-l_{u+1}}\left(l_{u}, l_{u+1} \gamma l_{u}-l_{u+1}-p\right) b\left(l_{u+1}, l_{u+1}-l_{l}+r_{t}+p\right) .
$$

Hence

$$
b\{(l),(r)\}=\sum_{p_{11}=1}^{l_{1}-l_{2}}\left(l_{1}, l_{2} \gamma l_{1}-l_{2}-p_{11}\right)\left[p_{11}\right],
$$

the term for $p_{11}=0$ vanishing.

Similarly,

$$
\begin{aligned}
& {\left[p_{11}\right]=\sum_{p_{12}=0}^{l_{2}-l_{3}} \sum_{p_{22}=1}^{l_{2}-l_{3}}\left(l_{2}, l_{3} \gamma l_{2}-l_{3}-p_{12}\right)\left(l_{2}, l_{3} \gamma l_{2}-l_{3}-p_{22}\right)\left[p_{11}+p_{12}, p_{22}\right]} \\
& =\Sigma\left|\begin{array}{ll}
\left(l_{2}, l_{3} \gamma l_{2}-l_{3}-p_{12}\right) & \left(l_{2}, l_{3} \gamma l_{2}-l_{3}-\left(p_{22}-p_{11}\right)\right) \\
\left(l_{2}, l_{3} \gamma l_{2}-l_{3}-p_{11}-p_{12}\right) & \left(l_{2}, l_{3} \gamma l_{2}-l_{3}-p_{22}\right)
\end{array}\right|\left[p_{11}+p_{12}, p_{22}\right]
\end{aligned}
$$


where the summation is taken over

$$
0 \leqslant p_{12} \leqslant l_{2}-l_{3}, \quad 1 \leqslant p_{22} \leqslant l_{2}-l_{3},
$$

subject to

$$
p_{11}+p_{12}>p_{22}>0 \text {. }
$$

We set

$$
P_{t u}=p_{t l}+p_{t, t+1}+\ldots+p_{t u} \text { when } t \leqslant u, \quad P_{t u}=0 \text { when } t>u,
$$

and use $\Sigma_{u}$ to denote summation over

$$
0 \leqslant p_{j u} \leqslant l_{u}-l_{u+1}, \quad \text { i.e. } \quad P_{j, u-1} \leqslant P_{j u} \leqslant l_{u}-l_{u+1}+P_{j, u-1},
$$

subject to

$$
P_{1 u}>P_{2 u}>\ldots>P_{u u}>0 \text {. }
$$

Then (5) may be written

$$
\left[P_{11}\right]=\Sigma_{2}\left|\left(l_{2}, l_{3} \gamma l_{2}-l_{3}+P_{\sigma 1}-P_{\tau 2}\right)\right|\left[P_{12}, P_{22}\right] \quad(\sigma, \tau=1,2) \text {. }
$$

Now $P_{1, u-1}>P_{s, u-1}$ for $s>1$, and hence from (4)

$$
\begin{aligned}
b\left(l_{u}, l_{u}-l_{t}+r_{i}+P_{s, u-1}\right) & \\
= & \sum_{w=0}^{l_{\mathrm{s}}-l_{u+1}+P_{1, u-1}}\left(l_{u}, l_{u+1} \gamma l_{u}-l_{u+1}+P_{s, u-1}-w\right) b\left(l_{u+1}, l_{u+1}-l_{t}+r_{t}+w\right) .
\end{aligned}
$$

Then by a proof similar to that of Theorem 1 we obtain

$$
\begin{aligned}
& {\left[P_{1, u-1}, P_{2, u-1}, \ldots, P_{u-1, u-1}\right]} \\
& \left.=\Sigma_{u} \mid\left(l_{u}, l_{u+1} \gamma l_{u}-l_{u+1}+P_{\sigma, u-1}-P_{\tau, u}\right)\right\}\left[P_{1 u}, P_{2 u}, \ldots, P_{u u}\right] \\
& \quad(\sigma, \tau=1,2, \ldots, u) .
\end{aligned}
$$

We find that if

$$
P_{1 u}>P_{2 u}>\ldots>P_{u u} \geqslant 0,
$$

then the coefficient of

$$
\left[P_{1 u}, P_{2 u}, \ldots, P_{u u}\right]
$$

in the expansion of (6) is

$$
\left|\left(l_{u}, l_{u+1} \gamma l_{u}-l_{u+1}+P_{\sigma, u-1}-P_{\tau, u}\right)\right| \quad(\sigma, \tau=1,2, \ldots, u) .
$$

Then in (8),

$$
P_{j, u-1}+l_{u}-l_{u+1} \geqslant P_{j u}
$$

otherwise the first $j$ elements of the last $n-j+1$ rows of (8) will be zero, and the determinant vanishes. Also

$$
P_{j u} \geqslant P_{j, u-1}
$$

otherwise the last $n-j+1$ elements of the first $j$ rows will be zero and the determinant vanishes. If $P_{u r}=0$, then rows $u$ and $u+1$ of (7) are equal. Hence the expansion of (6) follows. 
We set

$$
\begin{aligned}
& \xi_{\sigma}=l_{u}-l_{u+1}+P_{\sigma, u-1}+n+\sigma, \\
& \eta_{\tau}=P_{\tau, u}+n+\tau ;
\end{aligned}
$$

then

$$
\xi_{\sigma} \geqslant \xi_{\sigma+1} \text { and } \eta_{\tau} \geqslant \eta_{\tau+1}
$$

Hence

$$
\left|\left(l_{u}, l_{u+1} \gamma l_{u}-l_{u+1}+P_{\sigma, u-1}-P_{\tau, u}\right)\right|
$$

is the $S$-function $[1,110]$

$$
\{\tilde{\xi} / \tilde{\eta}\}
$$

of

$$
B_{l_{n+1}+1}, \ldots, \beta_{l_{u}-1}, \beta_{l u},
$$

where the tilde denotes conjugate partition.

$$
\text { Now } \quad\{\tilde{\xi} / \tilde{\eta}\}=\Sigma g_{\text {sn } \xi}\{\tilde{\zeta}\},
$$

where the $g_{\zeta n \xi}$ are non-negative integers determined by

$$
\{\zeta\}\{\eta\}=\Sigma g_{\zeta \eta \xi}\{\xi\}
$$

$[c f .11,110,91-96]$. Since $\{\tilde{\zeta}\}$ itself may be expanded as a polynomial in (10) with positive integral coefficients ${ }^{1}$, all the terms in the expansion of (6) as a polynomial in (10) have positive integral coefficients. Theorem 3 follows on repeated application of this argument.

We note that we may write in symbolic form:

$$
\begin{aligned}
& \begin{aligned}
\left|b\left(l_{s}, l_{s}-l_{t}+r_{t}\right)\right| \\
\quad=\prod_{u=1}^{n-1}\left\{\Sigma_{u}\left|\left(l_{u}, l_{u+1} \chi l_{u}-l_{u+1}+P_{\sigma, u-1}-P_{\tau, u}\right)\right|\left|b\left(l_{n}, l_{n}-l_{t}+r_{l}+P_{s, n-1}\right)\right|\right\} .
\end{aligned}
\end{aligned}
$$

1 This is well known [cf. 7]. Aitken [6] gives a direct proof that (9) may be expanded with positive integral coefficients.

\section{REFERENCES.}

1. D. E. Littlewood, Theory of Group Characters (Oxford, 1940).

2. K. A. Hirsch, "A note on Vandermonde's determinant", Journal London Math. Soc., $24(1949), 144-5$.

3. A. C. Aitken, Determinants and Matrices (5th ed., Edinburgh, 1948).

4. - "On determinants of symmetric functions", Proc. Edinburgh Math. Soc. (2), 1 (1929), 55.

5. __ " Note on dual symmetrie functions ", Proc. Edinburgh Malh. Soc. (2), 2 (1931), 164.

6. _- "The monomial expansion of determinantal symmetric functions", Proc. Royal Soc. Edinburgh (A), 61 (1941-3), 300-310.

7. O. H. Mitchell, " Note on determinants of powers", American Journal Math., 4 (1881), 341-4.

8. H. O. Foulkes, "Modified bialternants and symmetric function identities", Journal London Math. Soc., 25 (1950), 268-75.

\section{Department of Mathematios,}

UNIVERSITY OF ABERDEEN. 